\title{
In Vivo Mesenchymal Stem Cell Tracking with PET Using the Dopamine Type 2 Receptor and ${ }^{18}$ F-Fallypride
}

\author{
Veronika Schönitzer ${ }^{1}$, Florian Haasters ${ }^{1}$, Stefanie Käsbauer ${ }^{1}$, Veronika Ulrich ${ }^{1}$, Erik Mille ${ }^{2}$, Franz Josef Gildehaus ${ }^{2}$, \\ Janette Carlsen², Manuela Pape ${ }^{2}$, Roswitha Beck ${ }^{2}$, Andreas Delker ${ }^{2}$, Guido Böning ${ }^{2}$, Wolf Mutschler ${ }^{1}$, Wolfgang Böcker ${ }^{1}$, \\ Matthias Schieker ${ }^{1}$, and Peter Bartenstein ${ }^{2}$ \\ ${ }^{1}$ Department of Surgery, Experimental Surgery, and Regenerative Medicine, Ludwig-Maximilians-University Munich, Munich, \\ Germany; and ${ }^{2}$ Department of Nuclear Medicine, Ludwig-Maximilians-University Munich, Munich, Germany
}

\begin{abstract}
Human mesenchymal stem cells (hMSCs) represent a promising treatment approach for tissue repair and regeneration. However, little is known about the underlying mechanisms and the fate of the transplanted cells. The objective of the presented work was to determine the feasibility of PET imaging and in vivo monitoring after transplantation of dopamine type 2 receptor-expressing cells. Methods: An hMSC line constitutively expressing a mutant of the dopamine type 2 receptor (D2R80A) was generated by lentiviral gene transfer. D2R80A messenger RNA expression was confirmed by reverse transcriptase-polymerase chain reaction. Localization of the transmembrane protein was analyzed by confocal fluorescence microscopy. The stem cell character of transduced hMSCs was investigated by adipogenic and osteogenic differentiation. Migration capacity was assessed by scratch assays in time-lapse imaging. In vitro specific binding of ligands was tested by fluorescenceactivated cell sorting analysis and by radioligand assay using ${ }^{18} \mathrm{~F}-$ fallypride. Imaging of D2R80A overexpressing hMSC transplanted into athymic rats was performed by PET using ${ }^{18} \mathrm{~F}$-fallypride. Results: hMSCs showed long-term overexpression of D2R80A. As expected, the fluorescence signal suggested the primary localization of the protein in the membrane of the transduced cells. hMSC and D2R80A retained their stem cell character demonstrated by their osteogenic and adipogenic differentiation capacity and their proliferation and migration behavior. For in vitro hMSCs, at least 90\% expressed the D2R80A transgene and hMSC-D2R80A showed specific binding of ${ }^{18} \mathrm{~F}$-fallypride. In vivo, a specific signal was detected at the transplantation site up to $7 \mathrm{~d}$ by PET. Conclusion: The mutant of the dopamine type 2 receptor (D2R80A) is a potent reporter to detect hMSCs by PET in vivo.
\end{abstract}

Key Words: mesenchymal stem cells; in vivo imaging; cell tracking; dopamine 2 receptor; positron emission tomography

J Nucl Med 2014; 55:1342-1347

DOI: 10.2967/jnumed.113.134775

\section{A}

dult human mesenchymal stem cells (hMSCs) are mesodermal cells, which are able to migrate and to undergo differentiation into osteogenic, adipogenic, and chondrogenic lineages (1). Because

Received Nov. 12, 2013; revision accepted Jun. 2, 2014.

For correspondence contact: Florian Haasters, Department of Surgery, Experimental Surgery, and Regenerative Medicine, Ludwig-MaximiliansUniversity Munich, Nussbaumstrasse 20, 80336, Munich, Germany.

E-mail: florian.haasters@med.uni-muenchen.de

Published online Jul. 14, 2014.

COPYRIGHT (c) 2014 by the Society of Nuclear Medicine and Molecular Imaging, Inc. of these properties, hMSCs are particularly suitable for application in cell-based therapies (2-5).

Although preclinical in vivo studies demonstrated positive effects after transplantation of undifferentiated stem cells on the regeneration of various tissues such as bone, cartilage, and myocardium (6-8), the fate of these cells is not fully known.

Conventional histologic methods for detecting these cells have several limitations. They require invasive biopsies or euthanization to obtain various tissue samples of the animals at serial time points. To determine the efficacy of the implanted cells, noninvasive imaging methods are suited to answer 3 important questions: Are the cells migrating to the target tissue? Are the cells functional? Do the cells survive (9)?

The method of direct labeling requires incubation of the stem cells ex vivo with labeling agents, such as radiotracers. Afterward, the cells are transplanted into the target tissue or organ, and the imaging analysis is performed as shown recently for ${ }^{111}$ In-oxine labeling of hMSCs (10). Because of the dilution of the amount of radiotracer in dividing cells over time and the radioactive decay, this method is not suitable for measuring cell viability and is limited for long-term monitoring of stem cells (11). To evaluate the role of the transplanted hMSCs in fracture healing, the hMSCs must be observed over a longer time period than $2 \mathrm{~d}$. In addition, it is also critically important to ensure cell detection, viability, and function by the labeling procedure. An elegant way to achieve these goals is the stable transfection of hMSCs with a reporter gene encoding for an enzyme or a transmembrane receptor, which interacts with an exogenously given labeling agent (11). Advantageously, the reporter gene is passed on to the progeny cells and is expressed only in viable cells (12).

A suitable system is the transmembrane dopamine type 2 receptor $\left(D_{2} R\right)$. The wild-type $D_{2} R$ is not expressed in peripheral tissue, and the specific, high-affinity PET ligand ${ }^{18} \mathrm{~F}$-fallypride is available (13). Use of this ligand would allow monitoring of the location, magnitude, and duration of $\mathrm{D}_{2} \mathrm{R}$ gene expression by small-animal PET in living animals (14).

The binding of ligand on the wild-type $\mathrm{D}_{2} \mathrm{R}$ activates a G-proteinlinked signaling pathway that leads to a decrease in cellular cyclic adenosine monophosphate levels by inactivation of adenyl cyclase. To prevent this adverse effect on the cell physiology a mutant variant, the so-called D2R80A, was developed. Binding of ligands is completely uncoupled from the signaling pathway. Critically, the in vitro binding activity for ${ }^{3} \mathrm{H}$-spiperone and the in vivo binding activity for the used PET probe (3- $\left(2^{\prime}-{ }^{18} \mathrm{~F}\right.$-fluoroethyl)-spiperone) is essentially maintained, compared with the wild-type $\mathrm{D}_{2} \mathrm{R}$ (15). Signal detection using this method might be limited, because the receptor can bind 
only a single ligand molecule and can be blocked by nonlabeled endogenous ligands. Both may affect the sensitivity of this method (16-18).

The current study aimed to establish a system for monitoring hMSCs over a time period that is relevant for tissue healing. So we generated an hMSC line overexpressing D2R80A by lentiviral gene transfer. We examined whether the expression of the transgene affected the stem cell character after confirmation of the functionality of D2R80A in vitro. To assess the suitability of these cells for imaging in vivo, hMSC-D2R80A and control cells were injected into a muscle of the hind limb of athymic nude rats, and PET images were obtained on days 0,1 , and 7 after implantation.

\section{MATERIALS AND METHODS}

\section{Cloning of $D_{2} R$ and Transduction of HMSCs}

The original $\mathrm{pD} 2 \mathrm{R} 80 \mathrm{~A}$-internal ribosomal entry site-thymidine kinase (gly5)enhanced green fluorescent protein encoding for D2R80A was a kind gift of Sanjiv Sam Gambhir and of Andreas Jacobs and has been described elsewhere (19). The D2R80A fragment was restricted with BamHI and NheI (NEB) and blunt-ligated into the EcoRI Klenow (NEB) blunt-ended pENTR11 entry vector (Invitrogen). The insert was transferred into pLenti4.3/V5-DEST by homologous LR (Invitrogen) recombination. In parallel, a mock pLenti4.3/V5-DEST plasmid without expression cassette was created. The final plasmids pLenti4.3D2R80A and pLenti4.3-mock were verified by DNA sequencing. Lentivirus generation and transduction of hMSCs were performed as described elsewhere (20).

\section{Cell Culture and Characterization}

The well-established hTERT-immortalized hMSC line SCP-1 was used, and cells were cultured as described previously (20).

For analysis of cell growth, hMSC-mock and hMSC-D2R80A were observed over $20 \mathrm{~d}$, and the cell number was determined using a Neubauer counting chamber every third to fourth day. The experiments were performed in triplicate and repeated 3 times.

Cell proliferation was evaluated using WST-1 assay (Roche Diagnostics) according to the manufacturer's instructions. hMSCs $\left(0.6 \times 10^{3}\right.$ per well $)$ were cultured, and WST-1 reagent was applied after $36 \mathrm{~h}$. Two independent WST-1 assays were performed in triplicate.

Cell proliferation was analyzed with the Cell Proliferation ELISA, BrDU (colorimetric) kit (Roche) according to the manufacturer's instructions. hMSCs $\left(0.6 \times 10^{3}\right.$ per well) were seeded. After $12 \mathrm{~h}$, medium supplemented with $10 \mu \mathrm{M}$ 5-bromo-2'-deoxyuridine (BrDU) was added. BrDU incorporation was measured after $24 \mathrm{~h}$. Two independent experiments were performed in triplicate.

HMSC-mock and hMSC-D2R80A were seeded in 6-well plates and differentiated into the osteogenic and the adipogenic lineages as described previously (21). After $21 \mathrm{~d}$, Oil Red O staining and Alizarin Red staining were performed using a standard protocol. The experiments were repeated twice in triplicate.

The scratch assay was performed in 2 independent experiments as described previously (10). Obtained data were processed with AxioVision LE (Zeiss) and ImageJ software programs.

\section{Reverse Transcriptase-Polymerase Chain Reaction (RT-PCR) and Immunofluorescence}

For expression analysis on RNA level, total RNA was extracted from hMSC-D2R80A and hMSC-mock with the RNeasy Mini Kit (Qiagen) including the optional DNA digestion step, and $1 \mu \mathrm{g}$ of RNA was used for complementary DNA synthesis with an AMV kit (Invitrogen) according to the manufacturer's instructions. Semiquantitative RT-PCR was performed using Taq DNA Polymerase (Invitrogen) in an MG Research PCR machine (Bio-Rad). A 30-cycle standard PCR program with optimized annealing temperature for each primer pair (Table 1) was used.

For expression analysis on protein level, we used immunofluorescence. HMSC-mock and hMSC-D2R80A were fixed with $4 \%$ formaldehyde. After blocking with $3 \%$ bovine serum albumin, the cells were incubated with anti- $\mathrm{D}_{2} \mathrm{R}$ antibody $(5 \mu \mathrm{g} / \mathrm{mL}$; Millipore) and secondary Alexa Fluor 488-conjugated anti-rabbit antibody (Invitrogen). The cell nuclei were stained with propidium iodide (Sigma-Aldrich). Negative control was performed by omitting the primary antibody. Imaging was performed with a Zeiss laser scanning microscopy (LSM 510 Imager. Z1) microscope equipped with Plan-Apochromat $63 \times / 1.4$ Oil DIC objective. Alexa Fluor 488 and propidium iodide fluorescence were detected in dual-channel mode using appropriate filters. Experiments were repeated twice.

\section{Cell Binding Studies}

On the one hand, the activity of the $\mathrm{D} 2 \mathrm{R} 80 \mathrm{~A}$ receptor was studied in vitro using the $\mathrm{D}_{2} \mathrm{R}$ red antagonist ligand (Cisbio Bioassays). Therefore, $1 \times 10^{5}$ of hMSC-mock and hMSC-D2R80A were seeded. After $24 \mathrm{~h}$, the cells were washed with phosphate-buffered saline (PBS) and incubated with $12.5 \mathrm{nM}$ ligand in binding buffer for $3 \mathrm{~h}$. Afterward, cells were washed with PBS and finally resuspended. Stained cells were measured using a fluorescence-activated cell sorting (FACS) device (FACSCalibur; BD-Bioscience). Data were analyzed using FlowJo FACS analysis software 7.7 (Treestar Inc.). Five independent experiments were performed.

For the radioactive assays, ${ }^{18} \mathrm{~F}$-fallypride was synthesized using a SynChrom R\&D automatic synthesis module (Raytest) following a procedure previously described $(13,14)$ with slight modifications. The purity of the desired product was greater than $98 \%$ and the specific activity greater than $180 \mathrm{GBq} / \mu \mathrm{mol}$.

HMSC-D2R80A and hMSC-mock were cultured for $3 \mathrm{~d}$. Varying amounts of the labeled ${ }^{18} \mathrm{~F}$-fallypride were added (final activity concentration, 5-120 kBq/mL). After $2 \mathrm{~h}$, cells were harvested and washed twice with ice-cold PBS, and the cell-bound activity was determined by counting for ${ }^{18} \mathrm{~F}$ activity in a $\gamma$ counter. The counts were decay-corrected to the beginning of the incubation period. Each data point is a mean of 4 replicates.

\section{Tracking of Cells In Vivo and PET Imaging}

The animal procedure was in accordance with the principles for the care and use of laboratory animals of the Ludwig-MaximiliansUniversity of Munich and was approved by the Government Committee of Upper Bavaria. Ten male athymic nude rats (body weight, 250-300 $\mathrm{g}$; Harlan) were anesthetized with $2.0 \%-2.5 \%$ isoflurane in $2 \mathrm{~L}$ of $100 \%$ oxygen. For analgesia, the animals received meloxicam preoperatively $(0.2 \mathrm{mg} / \mathrm{kg}$ subcutaneously). Under sterile condition, rats were positioned in dorsal recumbency. hMSC-D2R80A $\left(2.4 \times 10^{7}\right)$ resuspended in $40 \mu \mathrm{L}$ of PBS was injected into a muscle of the hind limb using a 25gauge needle ( 7 times left; 3 times right). On the contralateral side, the same amount of hMSC-mock was inserted as negative control.

On days 0,1 , and 7 after transplantation, $20 \mathrm{MBq}$ of ${ }^{18} \mathrm{~F}$-fallypride were injected intravenously and PET image data subsequently recorded for 90 min on a small-animal PET scanner (Inveon DPET; Preclinical Solutions Siemens Healthcare Molecular Imaging). Dynamic 3-dimensional images with 17 frames $(5 \times 60,5 \times 180$, and $7 \times 600 \mathrm{~s})$ were reconstructed with corrections for photon attenuation and scattering as described previously (22) using 3-dimensional ordered-subset expectation maximization, followed by 32 maximum a posteriori 3-dimensional iterations with a zoom factor of 1.22 , resulting in a space-invariant spatial resolution of approximately $1.2 \mathrm{~mm}$. In these dynamic images, the location of the hMSC-D2R80A was detected visually by an experienced reader masked to the side and location of the injection. Afterward, standard volumes of interest (spheric with 3-mm diameter, 


\begin{tabular}{llcc}
\hline Target gene & \multicolumn{1}{c}{ Primer } & Annealing temperature $\left({ }^{\circ} \mathrm{C}\right)$ & PCR product \\
\hline D2R80A & Forward primer: 5'- CAAGCGCCGAGTTACTGTC & 58.8 & 334 bp \\
& Reverse primer: 5'- GCTGGTGCTTGACAGCATC & & \\
GAPDH & Forward primer: 5'- CAACTACATGGTTACATGTTC & 50 & 181 bp \\
& Reverse primer: 5'- GCCAGTGGACTCCACGAC & & \\
\hline
\end{tabular}

$\sim 29$ voxels) were placed around the site of focal ${ }^{18} \mathrm{~F}$-fallypride uptake at day 0 and in the contralateral thigh, and time-activity curves were derived. If the location of the hMSC-D2R80A was delineable at day 0 , this animal was included in further analysis and volumes of interest were placed in the images at day 1 and day 7 based on the anatomic information obtained at day 0 . Furthermore, a robust noninvasive 2-tissue-compartment kinetic model approach based on Logan plots was applied to quantitatively assess the $\mathrm{D}_{2} \mathrm{R}$ binding potential (BP), using the contralateral side as a reference tissue (23).

\section{Statistical Analysis}

Statistical evaluation was performed using the Prism software (version 5.02; GraphPad Software). Graphs and bar charts show mean values and $\mathrm{SD}$. An unpaired $t$ test was used for 2-group analysis. A $P$ value of less than 0.05 indicated evidence of significantly nonrandom occurrence.

\section{RESULTS}

\section{Expression of $D_{2} R$ Mutant in HMSCs}

The complementary DNA encoding a point-mutated form D2R80A was cloned into the lentiviral vector pLenti4.3V5-DEST containing a cytomegalovirus promoter for constitutive expression (Fig. 1A). Sequencing of the final construct pLenti4.3-D2R80A revealed that D2R80A exactly matched the database records. With D2R80A-lentivirus, it was possible to achieve a stable transduction of the SCP-1 hMSC line (20). In parallel, the cell line was infected with mock-lentivirus. Nontransduced cells were eliminated by antibiotic selection.

First, we confirmed D2R80A transgene expression on the messenger RNA level by semiquantitative RT-PCR (Fig. 1B).
No expression of D2R80A was observed in hMSC-mock. Next, we analyzed the localization of the transmembrane protein D2R80A in the cells by immunofluorescence (Fig. 1C). Confocal fluorescence microscopy verified the D2R80A expression on protein level. The receptor was detected in hMSC-D2R80A in filopodia or next to the cell edge. This staining pattern suggests that the transgene dopamine receptor is located in the membrane. No emission signal was detected in the negative control.

\section{Stem Cell Character of D2R80A-Overexpressing HMSCs}

Differentiation of HMSC-D2R80A and hMSC-mock cells into adipogenic and osteogenic lineages was confirmed by Oil Red $\mathrm{O}$ and Alizarin Red staining, respectively (Figs. 2A and 2B).

The D2R80A-overexpressing cells showed a slightly lower proliferation rate than the hMSC-mock, but both cell lines entered the plateau phase after approximately $15 \mathrm{~d}$ (Fig. 2C). These results were confirmed measuring DNA synthesis by incorporation of BrDU (Fig. 2D) and determining overall metabolic activity by cleavage of WST-1 (Fig. 2E). The cell growth of the hMSCD2R80A is nonsignificantly reduced, compared with hMSCmock.

In scratch assay analyses, the closure of an artificial wound in a confluent cell layer was determined. After $12.5 \mathrm{~h}, 63.5 \% \pm 3.6 \%$ in the hMSC-mock and $62.0 \% \pm 4.6 \%$ in the hMSC-D2R80A of the artificial wound were closed; after $25 \mathrm{~h}$, the SCP1-D2R80A closed $95.8 \% \pm 2.2 \%$ of the scratch and the hMSC-mock closed $95.5 \% \pm 1.8 \%$ (Fig. 2F).

\section{In Vitro Activity of Transgene D2R80A}

The ability of the transduced cells to bind specifically ligands of the dopamine receptor was tested in 2 different assays. FACS analysis showed that $90 \%$ of hMSC-D2R80A cells were labeled with the $\mathrm{D}_{2} \mathrm{R}$ red antagonist using hMSC-mock as a negative control (Fig. 3A).

Radioactivity assays showed that hMSCD2R80A could be specifically labeled with the radioactive ligand in different concentrations, whereas only background signal was observed in hMSC-mock (Fig. 3B). At the addition of $30 \mathrm{~Bq} / \mathrm{mL}$, we already measured the maximal binding activity of approximately $22 \mathrm{mBq} /$ cell.

\section{In Vivo Imaging of D2R80A- \\ Overexpressing Cells}

Before all in vivo experiments, a receptor expression of at least $90 \%$ of hMSC-D2R80A was confirmed by a D2R red antagonist binding assay. 


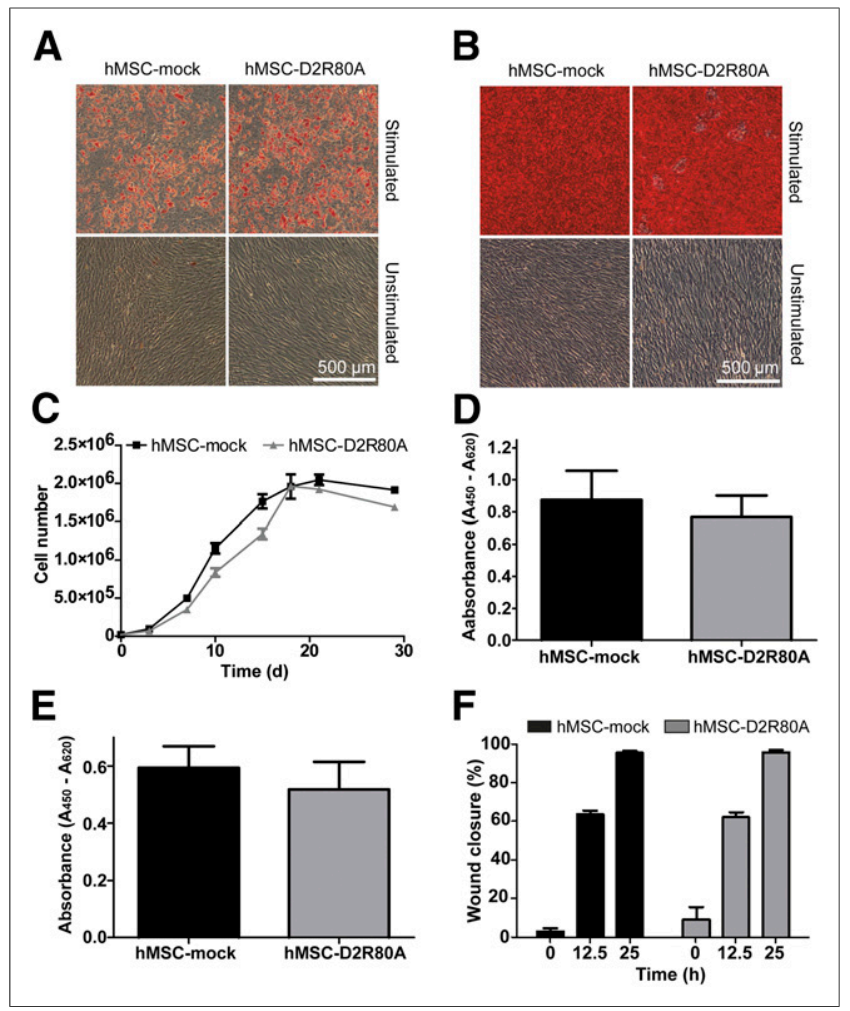

FIGURE 2. Analysis of stem cell character of hMSC-D2R80A. (A) During adipogenic differentiation, formed lipid vacuoles were visualized by Oil Red O staining. (B) Osteogenic differentiation was confirmed by Alizarin Red staining. (C) Growth curves of hMSC-D2R80A and hMSCmock. (D and E) Cell proliferation of hMSC-D2R80A and hMSC-mock measured by incorporation of BrDU (D) and WST-assay (E). (F) Scratch assays show closure of original scratch area in percentage after 12.5 and $25 \mathrm{~h}$. Bars present means $\pm \mathrm{SD}$.

Because of the enzymatic defluorination of fallypride occurring in rodents, but not to a relevant extent in humans, the bones gradually absorbed ${ }^{18} \mathrm{~F}$, resulting in visualization of the skeleton (22).

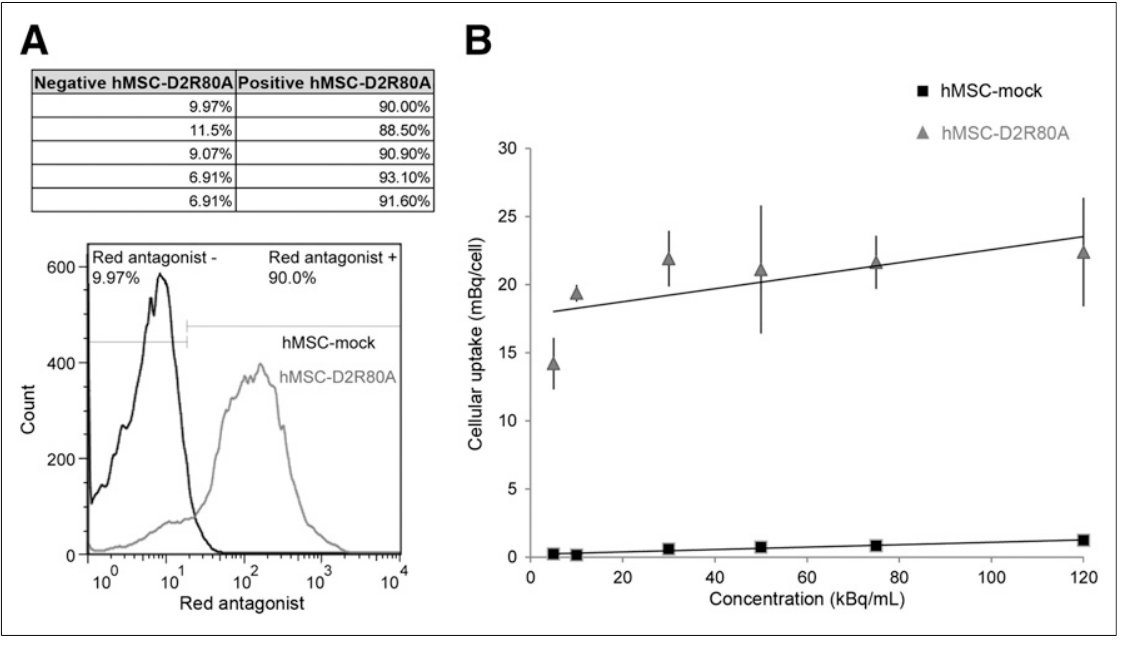

FIGURE 3. Investigation of D2R80A activity by ligand binding assays. (A) Approximately $90 \%$ of hMSC-D2R80A were able to bind D2R red antagonist shown by FACS analysis. Results of 5 repetitions of experiment are listed in table. (B) hMSC-D2R80A were specifically labeled by incubation with ${ }^{18} \mathrm{~F}$-fallypride.
At the injection site of hMSC-mock, only background signal was detected in all animals at any time point investigated (Fig. 4A). In 1 animal, the location of the hMSC-D2R80A could not be reliably determined at day 0 because of a low tracer uptake. The specific signal-to-background ratio was below 1.2, and the BP was below 0.5 . In a second animal, the PET scan at day 1 failed because of technical problems. Both animals were therefore excluded, and all further analyses were performed with the remaining 8 animals.

In 6 of these 8 animals, a signal-to-background ratio above 1.2 could be followed up to day 7 .

Because there was no observable uptake of fallypride at any time on the contralateral side where hMSC-mock was administered, the signal consistently observed on the side of hMSCD2R80A is very likely to reflect specific binding. Furthermore, the kinetics of fallypride were clearly distinguishable from the unspecific bone uptake. Figure 4B presents the average ratios of fallypride accumulations for the 8 animals at the hMSC-D2R80A and contralateral hMSC-mock location over time, obtained at days 0,1 , and 7 . These data suggest a pseudoequilibrium between 30 and $60 \mathrm{~min}$ after injection, where mean signal-to-background ratios between the MSCs and the contralateral side were $2.2 \pm 0.6$ on day $0,2.2 \pm 0.7$ on day 1 , and $1.8 \pm 0.6$ on day 7 . The maximum ratio observed in 1 measurement was 3.5 on day $0,3.7$ on day 1 , and 2.6 on day 7. The modeling of tracer kinetics yielded a mean BP of $1.1 \pm 0.5$ (maximum, 2.1) on day $0,1.1 \pm 0.6$ (maximum, 1.9) on day 1 , and $0.8 \pm 0.5$ (maximum, 1.5) on day 7 .

\section{DISCUSSION}

hMSCs are promising candidates for a cell-based therapy of challenging large bone defects and severe surrounding soft-tissue damage (24). Several phase II trials using hMSCs to improve tissue regeneration presented promising results. Designated transplant centers already routinely use hMSCs on the basis of these data (25). Although hMSCs are crucial for the induction of repair processes, their behavior in vivo with regard to survival, migration to the site of injury, and fate is almost unknown (26). Therefore, in vivo cell tracking of hMSCs is important to further understand the mechanisms and dynamics of repair processes induced by these cells. So far, only few attempts have been made to monitor the contribution of hMSC to bone and softtissue healing or regeneration in vivo (10). Only very few studies using reporter gene systems focus on hMSCs and on musculoskeletal tissue. PET imaging of hMSCs expressing herpes simplex virus type 1 thymidine kinase (HSV1-Tk) implanted subcutaneously was recently described in the literature (27). In contrast, monitoring of HSV1-TK-expressing hMSCs in a 0.5mm-sized bone defect could not be established (28).

Other approaches in molecular imaging are based on bioluminescence of proteins such as luciferase (Luc). Recently, hMSCs overexpressing firefly Luc could be detected in an orthotopic mouse brain human tumor model (29). The limited light penetration through the tissue results in a relatively poor 


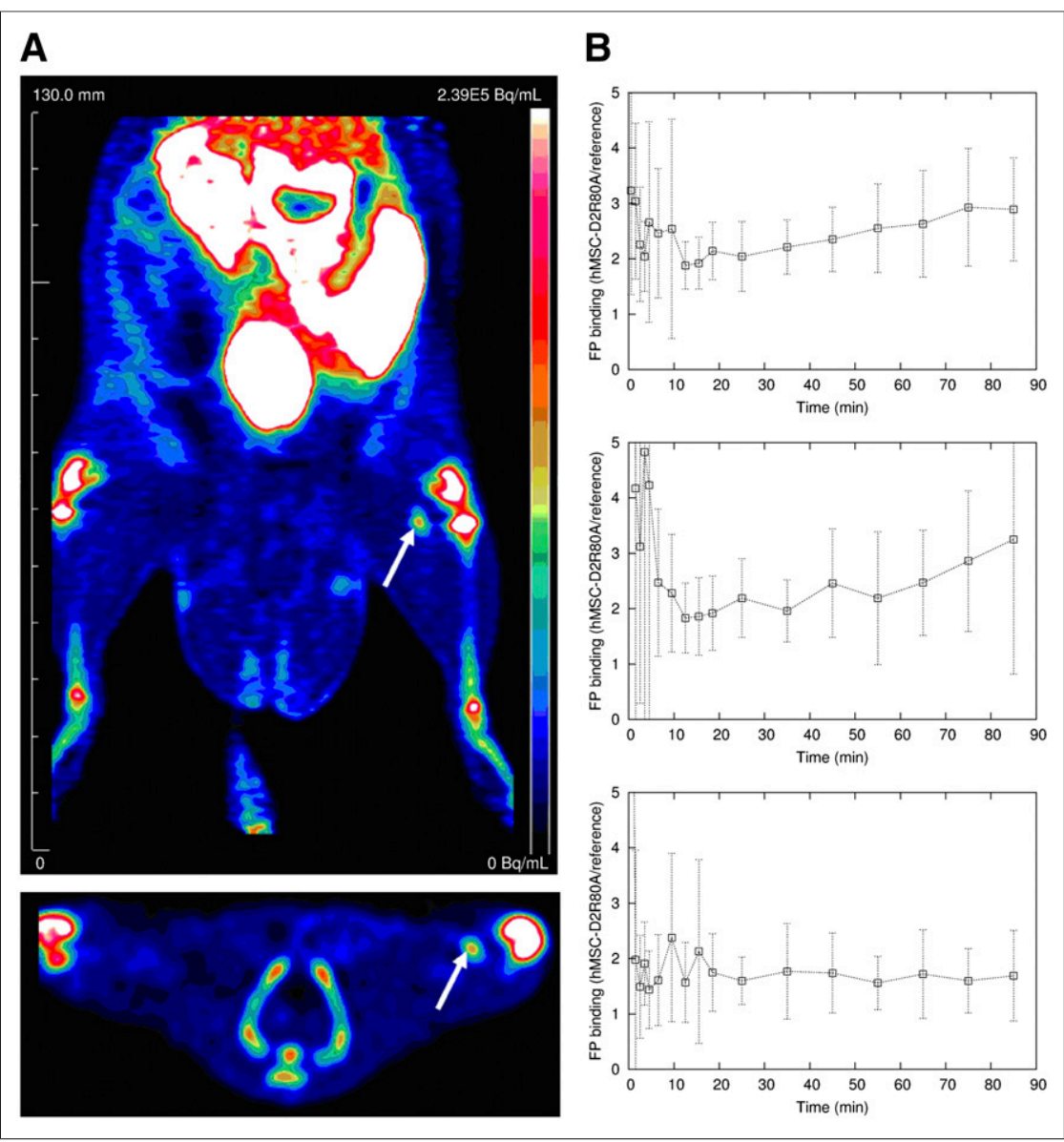

FIGURE 4. Cell tracking of hMSC-D2R80A. (A) PET images of 1 representative animal at day 1. Arrows mark signal at injection site of hMSC-D2R80A. Only background signal is the theoretic location of hMSC-mock at contralateral side. (B) Specific binding of fallypride at hMSC-D2R80A vs. hMSC-mock location over time averaged for 8 positive animals at day 0 (top), day 1 (middle), and day 7 (bottom). Error bars indicate SD. FP $={ }^{18} \mathrm{~F}$-fallypride.

spatial resolution. Therefore, bioluminescence imaging can effectively be used in small-animal models only.

Molecular imaging methods using MR imaging reporters allow high spatial resolution but lack sensitivity, compared with radionuclide imaging reporters (12). Most reporter gene systems for MR imaging rely on negative contrast, which can be difficult to detect in a heterogeneous image. So far, these genes have not been used in hMSCs.

At the site of injury, hMSCs are an important but rare cell fraction. Therefore, an imaging system with high sensitivity is needed for hMSC detection. A reporter gene system using D2R80A-overexpressing hMSC and small-animal PET combines the advantage of high sensitivity and high spatial resolution. Furthermore, this system holds promise for molecular imaging of the musculoskeletal system in humans, since $\mathrm{D}_{2}$ Rs are physiologically expressed in human brain only (13).

Using lentiviral vectors, which are known to be suited for stable transduction of foreign genes with high efficiency in hMSCs (30), we achieved stable integration of D2R80A into the genome of immortalized hMSCs. The hMSC-D2R80A retained their stem cell character demonstrated by their osteogenic and adipogenic differentiation capacity as well as their proliferation and migration behavior (Fig. 2). Furthermore, hMSC-D2R80A were capable of binding 2 specific ligands in vitro, confirming the expression of the transmembraneous D2R80A (Fig. 3). These results go well in line with the findings of Aung et al. on $\mathrm{D}_{2} \mathrm{R}$-expressing HeLa-Tet-On cells (31).

In our in vivo studies we were able to clearly detect the transplanted hMSCD2R80A in the hind limb (Fig. 4A). The time-activity curves and the results of the kinetic modeling suggest a specific binding of ${ }^{18}$ F-fallypride at the receptor (Fig. 4B). Accordingly, the hMSC-D2R80A survive in vivo, feature the favored ligand properties over time, and thereby represent a reliable model for long-term cell tracking of hMSC.

In our model, we administered on average $2.4 \times 10^{7} \mathrm{hMSC}-\mathrm{D} 2 \mathrm{R} 80 \mathrm{~A}$ and were able to measure a target-to-background ratio in the muscle (under optimal conditions) of around 3.5 to 1 . Assuming that a target-to-background ratio of $1.2-1$ is enough to reliably detect a signal, it can be supposed that the detection limit based on the performance characteristics of our camera system lies at around $5 \times 10^{6}$ administered cells (32). This is to the similar order of magnitude reported for other genetically marked stem cells (33).

Although we demonstrated that the proposed approach using hMSC-D2R80A and the high-affinity ligand ${ }^{18} \mathrm{~F}$-fallypride is suited for monitoring stem cells in concentrations reflecting the therapeutic situation, at least in rodents dehalogenation limits the use of ${ }^{18} \mathrm{~F}$ fallypride for the monitoring of hMSCs in bone (22). In this situation an ${ }^{11} \mathrm{C}$-labeled ligand with similar affinity (e.g., ${ }^{11} \mathrm{C}-(+)-4$-propyl-9hydroxynaphthoxazine) should be used (34).

The nonspecific uptake of ${ }^{18} \mathrm{~F}$-fallypride in the bowel and liver limits the use of this system in the abdomen. There is unfortunately no advantage here over, for example, the use of hMSCs expressing HSV1-Tk, which shows even a more intense bowel uptake (35). Because we observed low cardiac and pulmonary ${ }^{18}$ F-fallypride uptake, the tracking of D2R80A-expressing hMSC in these organs seems possible and will be a target of further research.

Because ${ }^{18} \mathrm{~F}$-fallypride binding in the human brain is well understood, there is also a potential to use our established lentiviral system for monitoring hMSCs in patients. Former studies on gene therapy with the first generation of retroviral vector insertions near oncogenes reported leukemia and myelodysplasia in some patients. However, Biffi et al. (36) and Aiuti et al. (37) reported promising results from 2 gene therapy trials for the treatment of neurodegenerative disorders and immunodeficiency diseases in children. The researchers also used lentiviral vectors, which showed no favored integrations in a particular gene locus, thus decreasing the likelihood of activating oncogenes. Therefore, we presume that the third generation of lentiviral vectors used in our study provides a safe method for a future human application.

To the best of or knowledge, there is no hint that the $\mathrm{D}_{2} \mathrm{R}$ might act as a suicide gene such as the HSV1-Tk (38). However, because 
the used variant D2R80A is not functional and lentiviral vectors have low genotoxicity (20), the hMSC-D2R80A should have no potential for malignant transformation. Therefore, a suicide gene therapy should not be necessary. Unfortunately, the used $D_{2} R$ gene and its variants are rat genes. For their use in clinical studies the human $D_{2} R$ equivalents will have to be developed, to prevent a possible immune response (16-18).

\section{CONCLUSION}

Understanding the destiny of transplanted stem cells is crucial for the development of stem cell therapeutics. Noninvasive imaging is an ideal method for stem cell tracking. In this study, we demonstrated that the combination of D2R80A as a reporter labeled with ${ }^{18} \mathrm{~F}$-fallypride is feasible for detection and follow-up of hMSCs by small-animal PET.

\section{DISCLOSURE}

The costs of publication of this article were defrayed in part by the payment of page charges. Therefore, and solely to indicate this fact, this article is hereby marked "advertisement" in accordance with 18 USC section 1734. This work was supported in part by a research grant from the Bavarian Research Foundation (Bavarian Research Collaboration for cell-based regeneration in senescence [www.forzebra.de]). No other potential conflict of interest relevant to this article was reported.

\section{REFERENCES}

1. Caplan AI, Bruder SP. Mesenchymal stem cells: building blocks for molecular medicine in the 21st century. Trends Mol Med. 2001;7:259-264.

2. Drosse I, Volkmer E, Capanna R, De Biase P, Mutschler W, Schieker M. Tissue engineering for bone defect healing: an update on a multi-component approach. Injury. 2008;39(suppl 2):S9-S20.

3. Kassem M. Stem cells: potential therapy for age-related diseases. Ann N Y Acad Sci. 2006; 1067:436-442.

4. Pittenger MF, Mackay AM, Beck SC, et al. Multilineage potential of adult human mesenchymal stem cells. Science. 1999;284:143-147.

5. Prockop DJ. Marrow stromal cells as stem cells for nonhematopoietic tissues. Science. 1997;276:71-74.

6. Bruder SP, Jaiswal N, Ricalton NS, Mosca JD, Kraus KH, Kadiyala S. Mesenchymal stem cells in osteobiology and applied bone regeneration. Clin Orthop Relat Res. 1998; (355, suppl):S247-S256.

7. Schantz JT, Hutmacher DW, Lam CX, et al. Repair of calvarial defects with customised tissue-engineered bone grafts II: evaluation of cellular efficiency and efficacy in vivo. Tissue Eng. 2003;9(suppl 1):S127-S139.

8. Xian CJ, Foster BK. Repair of injured articular and growth plate cartilage using mesenchymal stem cells and chondrogenic gene therapy. Curr Stem Cell Res Ther. 2006;1:213-229.

9. Massoud TF, Gambhir SS. Molecular imaging in living subjects: seeing fundamental biological processes in a new light. Genes Dev. 2003;17:545-580.

10. Gildehaus FJ, Haasters F, Drosse I, et al. Impact of indium-111 oxine labelling on viability of human mesenchymal stem cells in vitro, and 3D cell-tracking using SPECT/CT in vivo. Mol Imaging Biol. 2011;13:1204-1214.

11. Rodriguez-Porcel M. In vivo imaging and monitoring of transplanted stem cells: clinical applications. Curr Cardiol Rep. 2010;12:51-58.

12. Kircher MF, Gambhir SS, Grimm J. Noninvasive cell-tracking methods. Nat Rev Clin Oncol. 2011;8:677-688.

13. Siessmeier T, Zhou Y, Buchholz HG, et al. Parametric mapping of binding in human brain of D2 receptor ligands of different affinities. J Nucl Med. 2005;46: 964-972.
14. Rominger A, Wagner E, Mille E, et al. Endogenous competition against binding of $\left[{ }^{18} \mathrm{~F}\right] \mathrm{DMFP}$ and $\left[{ }^{18} \mathrm{~F}\right]$ fallypride to dopamine $\mathrm{D}_{2 / 3}$ receptors in brain of living mouse. Synapse. 2010;64:313-322.

15. Liang Q, Satyamurthy N, Barrio JR, et al. Noninvasive, quantitative imaging in living animals of a mutant dopamine D2 receptor reporter gene in which ligand binding is uncoupled from signal transduction. Gene Ther. 2001;8:1490-1498.

16. Brader P, Serganova I, Blasberg RG. Noninvasive molecular imaging using reporter genes. J Nucl Med. 2013;54:167-172.

17. Serganova I, Ponomarev V, Blasberg RG. Radionuclide-based reporter gene imaging: pre-clinical and clinical implementation and application. Nucl Med Rev. 2012;15(suppl C):C20-C36.

18. Serganova I, Ponomarev V, Blasberg R. Human reporter genes: potential use in clinical studies. Nucl Med Biol. 2007;34:791-807.

19. Kummer C, Winkeler A, Dittmar C, et al. Multitracer positron emission tomographic imaging of exogenous gene expression mediated by a universal herpes simplex virus 1 amplicon vector. Mol Imaging. 2007;6:181-192.

20. Böcker W, Yin Z, Drosse I, et al. Introducing a single-cell-derived human mesenchymal stem cell line expressing hTERT after lentiviral gene transfer. $J$ Cell Mol Med. 2008;12:1347-1359.

21. Böcker W, Rossmann O, Docheva D, Malterer G, Mutschler W, Schieker M. Quantitative polymerase chain reaction as a reliable method to determine functional lentiviral titer after ex vivo gene transfer in human mesenchymal stem cells. J Gene Med. 2007;9:585-595.

22. Mille E, Cumming P, Rominger A, et al. Compensation for cranial spill-in into the cerebellum improves quantitation of striatal dopamine $D_{2 / 3}$ receptors in rats with prolonged $\left[{ }^{18} \mathrm{~F}\right]$-DMFP infusions. Synapse. 2012;66:705-713.

23. Ichise M, Ballinger JR, Golan $\mathrm{H}$, et al. Noninvasive quantification of dopamine D2 receptors with iodine-123-IBF SPECT. J Nucl Med. 1996;37:513-520.

24. Bruder SP, Fink DJ, Caplan AI. Mesenchymal stem cells in bone development, bone repair, and skeletal regeneration therapy. J Cell Biochem. 1994;56:283-294.

25. Keating A. Mesenchymal stromal cells: new directions. Cell Stem Cell. 2012;10: 709-716.

26. Chamberlain G, Fox J, Ashton B, Middleton J. Concise review: mesenchymal stem cells-their phenotype, differentiation capacity, immunological features, and potential for homing. Stem Cells. 2007;25:2739-2749.

27. Love Z, Wang F, Dennis J, et al. Imaging of mesenchymal stem cell transplant by bioluminescence and PET. J Nucl Med. 2007;48:2011-2020.

28. Lee SW, Padmanabhan P, Ray P, et al. Stem cell-mediated accelerated bone healing observed with in vivo molecular and small animal imaging technologies in a model of skeletal injury. J Orthop Res. 2009;27:295-302.

29. Menon LG, Pratt J, Yang HW, Black PM, Sorensen GA, Carroll RS. Imaging of human mesenchymal stromal cells: homing to human brain tumors. J Neurooncol. 2012;107:257-267.

30. Van Damme A, Thorrez L, Ma L, et al. Efficient lentiviral transduction and improved engraftment of human bone marrow mesenchymal cells. Stem Cells. 2006;24:896-907.

31. Aung W, Okauchi T, Sato M, et al. In-vivo PET imaging of inducible D2R reporter transgene expression using $\left[{ }^{11} \mathrm{C}\right] \mathrm{FLB} 457$ as reporter probe in living rats. Nucl Med Commun. 2005;26:259-268.

32. Chatziioannou AF, Cherry SR, Shao Y, et al. Performance evaluation of microPET: a high-resolution lutetium oxyorthosilicate PET scanner for animal imaging. J Nucl Med. 1999;40:1164-1175.

33. Pei Z, Lan X, Cheng Z, et al. A multimodality reporter gene for monitoring transplanted stem cells. Nucl Med Biol. 2012;39:813-820.

34. Gallezot JD, Beaver JD, Gunn RN, et al. Affinity and selectivity of $\left[{ }^{11} \mathrm{C}\right]-(+)-$ PHNO for the D3 and D2 receptors in the rhesus monkey brain in vivo. Synapse. 2012;66:489-500.

35. Hung SC, Deng WP, Yang WK, et al. Mesenchymal stem cell targeting of microscopic tumors and tumor stroma development monitored by noninvasive in vivo positron emission tomography imaging. Clin Cancer Res. 2005;11:7749-7756.

36. Biffi A, Montini E, Lorioli L, et al. Lentiviral hematopoietic stem cell gene therapy benefits metachromatic leukodystrophy. Science. 2013;341:1233158.

37. Aiuti A, Biasco L, Scaramuzza S, et al. Lentiviral hematopoietic stem cell gene therapy in patients with Wiskott-Aldrich syndrome. Science. 2013;341: 1233151.

38. Duarte S, Carle G, Faneca H, de Lima MC, Pierrefite-Carle V. Suicide gene therapy in cancer: where do we stand now? Cancer Lett. 2012;324:160-170. 